\title{
NBS-LLR Marker Assisted Screening of Resistance Genotypes for Mungbean Yellow Mosaic Virus (MYMV) in Mungbean (Vigna radiata (L.) Wilcezk) Genotypes
}

\author{
N. Jyothi" ${ }^{*}$ B. R. Patil, Ramesh Bhat and B. M. Lokesh Kumar
}

Department of Genetics and plant breeding, University of agriculture science, Dharwad, Karnataka, India

*Corresponding author

\section{A B S T R A C T}

\begin{tabular}{|l|}
\hline Ke y w o r d s \\
NBS-LLR, \\
mungbean, \\
$\begin{array}{l}\text { Mungbean yellow } \\
\text { mosaic virus } \\
\text { MYMV }\end{array}$ \\
\hline Article Info \\
\hline $\begin{array}{l}\text { Accepted: } \\
\text { 22 July 2020 } \\
\text { Available Online: } \\
\text { 10 August } 2020\end{array}$ \\
\hline
\end{tabular}

Mungbean is one of the most important pulse crops which is native to India. The yield of mungbean has been stagnant over years. Improvement in yield of mungbean is becoming difficult mainly due to the occurrence of pest and diseases. Among the various diseases Mungbean yellow mosaic virus (MYMV), which is a Begomo virus transmitted through white fly, Bemesia tabaci, causing significant yield losses in mungbean, leading to a yield loss. With this aspect the present study was carried to identify the resistance source employing NBS-LLR markers. A total of fifty-five NBS-LLR markers was screened in fifty-four genotypes of mungbean. Out of fifty-five markers seven polymorphic viz. XLRR, RGA-1TG, MTB-99, S1, CLRR-INV1, ptokiniIN and VURS02F16V markers were identified these markers were specifically linked with MYMV disease and also twenty-one resistance genotypes were observed these resistance lines can be further used for resistance breeding programme.

\section{Introduction}

Mungbean (Vigna radiata (L.) Wilcezk) is also known as green gram, is one of the important pulse crop India. It belongs to the family Leguminoceae having the chromosome number $2 \mathrm{n}=22$, is warm season annual and self-pollinated crop. Currently, the global annual growing and production is about 6 million hectares worldwide and global 3 million tonnes, respectively. India leads the production of mungbean worldwide followed by China and Myanmar Nair et al., (2014). In India, mungbean is grown on an area of about 3 million hectares with the production of about 1 million tonnes. The major mungbean grown states are Orissa, Maharashtra, Andhra Pradesh, Telangana, Rajasthan, Madhya Pradesh, Bihar, Karnataka, and Uttar Pradesh. It is an excellent and inexpensive source of vegetable protein and ranks high among the different pulse crops, grown in India. Mungbean contains about $23.9 \%$ protein; rich in lysine which is generally low or deficient in cereals. Mature seeds are rich in proteins, and cooked seeds form a valuable constituent of diet of considerable number of people in country. 
The tender pods of mungbean are also eaten as vegetable. The ripe seeds serve as a source of pulse which is an important constituent of diet in Indian subcontinent.

Several fungal and viral diseases are reported which caused severe reduction inmungbean and urdbean yield Paul et al., (2013). The mungbean yellow mosaic virus (MYMV) is the most devastating, especially in South Asian countries. MYMV can cause yield loss of about 75-100 per cent depending on disease incidence, virus strains, mungbean genotypes and interaction between these factors (Singh, 1980). MYMV is caused by different species of Begomovirus (family Geminiviridae these viruses are transmitted by whitefly (Bemisiatabaci).

The management of MYMV is focused mainly on whitefly control. However pesticides can provide temporary management of whiteflies, but do not give effective control of MYMV. A more efficient and environmentally safe long-term solution is the development of mungbean cultivars resistant to both virus and its vector Bemisia tabaci. Therefore, using resistant varieties is the most desirable means of managing the disease. For better identification of desired genotype in the breeding programme, there is a need to identify DNA markers linked to mungbean yellow mosaic virus (MYMV) in mungbean. Among the various molecular markers that are being used, the use of Resistant Gene Analogues and Resistant Gene Homologues is very efficient as they originate from the NBS-LRR disease resistant motifs and they can be conveniently designed from diagnostic motifs of known disease resistant genes (Kanazin et al., 1996; Huang and Gill 2001; Yan et al., 2003). Resistant genes (RGenes) are genes in plant genomes that convey plant disease resistant against pathogens by producing $\mathrm{R}$ proteins. These large, abundant proteins are involved in the detection of diverse pathogens, including bacteria, viruses, fungi, nematodes, insects and oomycetes. With this background knowledge, the main aim of the present study was carried out to identify the resistance source for the resistance breeding programme.

\section{Materials and Methods}

\section{Plant material}

A total of fifty four mungbean (Table 1), used in this study were raised in Department of Genetics and plant breeding, University of agriculture science. Dharwad.

\section{DNA extraction}

All fifty-five genotypes of mungbean were sown in sowing trays which contained a mixture of coirepith and sand. When the plants were at two leaf stage, the DNA was isolated using a modified Saghai and Maro of (1984) CTAB method. Grind tissue with liquid nitrogen in a micro centrifuge tube. When liquid nitrogen has all sublimed away, add hot (65 o C) CTAB buffer (the volume of the buffer added should be approximately equal to the volume of the tissue. The mixture should now resemble thick, slimy soap) then incubated it for $15-20 \mathrm{~min}$ at $65 \mathrm{oC}$ followed by cooling the tubes at room temperature. Then Centrifuge the tubes at 13,000 rpm for 15 min. Take out the supernatant add equal volume of Chloroform and Iso amyl alcohol (24:1) with gentle mixing. Again centrifuge the tubes at 13,000 rpm for $15 \mathrm{~min}$. repeat the steps of CI treatment twice. Take supernatant and add twice amount of Iso Propanol. Keep for overnight incubation at -20 o $\mathrm{C}$. After 24hthe tubes were centrifuged at 10,000 rpm for $10 \mathrm{~min}$ at cool condition. After the centrifuge supernatant was discarded followed by ethanol wash to pellet at 8000 rpm for $5 \mathrm{~min}$ and repeat the same procedure 2-3 times. Finally pellet were draied the at 
room temperature for 2-3 hrs then dissolve the pellet in T $10 \mathrm{E} 1$ (based on the pellet obtained) Store at -20 o C. the DNA was purified from RNA by treating with RNAse (Bangalore Genei). The quality of DNA was assessed by taking Nano drop readings and concentration of the DNA was adjusted.

\section{NBS-LRR assay}

A standard PCR reaction were performed in $20.00 \mu \mathrm{l}$ volume containing $2.0 \mu \mathrm{l}$ of $15 \mathrm{ng}$ of template DNA, 3 units $/ \mu \mathrm{l}$ of Taq DNA polymerase (Bangalore Genei Ltd., Bangaluru, Karnataka, India) $2.00 \mu \mathrm{l}$ of $10 \mathrm{X}$ of assay buffer, $2.5 \mathrm{mM}$ of $2 \mu$ ldNTPs, $1 \mu \mathrm{l}$ of $0.5 \mu \mathrm{M}$ each of forward and reverse primers was performed in thermocycler (Eppendoff) with the following PCR conditions: DNA denaturation at $95{ }^{\circ} \mathrm{C}$ for $74 \mathrm{~min}, 35$ cycles of $95{ }^{\circ} \mathrm{C}$ for $1 \mathrm{~min}$, primer annealing at $50-58{ }^{\circ} \mathrm{C}$ for 30 s and a primer extension step of $72{ }^{\circ} \mathrm{C}$ for $1 \mathrm{~min}$ and final elongation step was extended to $72{ }^{\circ} \mathrm{C}$ for $7 \mathrm{~min}$. Further the PCR products $(20 \mu \mathrm{l})$ were subjected to Electrophoresis on 3 per cent Agarose gel in 1X TAE buffer for 3 hours at 50 volts. A 100 bp ladder (Bangalore Genei) was used as a known standard size marker. The electronic image of ethidium bromide stained gels was captured using UVITEC Cambridge Doc.

\section{Recording of the observations}

Presence of a band was marked as "+" and absence of band was marked as "-". The allele sizes were determined by comparing with the $100 \mathrm{bp}$ marker.

\section{Statistical method of analysis}

Allelic variation was calculated from the frequencies of genotypes at each locus as the polymorphic information content. Genetic parameters namely frequency of the abundant allele, genotype frequency and polymorphic information content (PIC) were estimated using the software program Power Marker version 3.25 (Liu and Muse, 2005).

\section{Results and Discussion}

\section{PCR amplification}

PCR approach was chosen as a first step in the identification of putative resistant genes in mungbean. A set of fifty-five Resistant Gene Homologous (RGHs) from cowpea (Vigna unguiculata), chickpea (Ciceraritinum), Medicago truncatula and were screened across fifty-five genotypes of green gram. Increasing the stringency of PCR condition by adopting higher annealing temperatures and "touch-down" protocols were not successful in obtaining specific amplicon, hence it was necessary to optimize the PCR conditions. The optimum annealing temperature, determined after testing the temperatures through gradient PCR approach, ranged between $43^{\circ} \mathrm{C}$ and $53^{\circ} \mathrm{C}$ (Table 3). Majority of primers which belonged to $\mathrm{Vu}$ series derived from NBS-LRR disease resistant motifs, generated amplification at $47^{\circ} \mathrm{C}$ and above, while the remaining primers produced amplification between $43^{\circ} \mathrm{C}$ and $49^{\circ} \mathrm{C}$ (Fig. $1)$.

\section{Study NBS-LRR markers and study the polymorphism in the black gram and green gram genotypes}

Specificity to resistance among a total of 54 genotypes of green gram was examined with 55 markers. Only 32 markers out of 55 amplified successfully in all the fifty four genotypes with twenty markers producing no amplification. Out of thirty two markers which produced amplification only seven markers generated polymorphism, remaining twenty-five were monomorphic. The seven polymorphic markers collectively yielded 15 alleles in green gram with an average of 2.1 
polymorphic alleles per locus respectively. The characterization of these 15 polymorphic markers is provided in Table 2. Markers
XLRR, Pto kin1, S1-INV, S1 and Pto-kin1IN produced three alleles while reaming markers produced two alleles each.

Table.1 Details of Mungbean/green gram genotypes used in the study

\begin{tabular}{|c|c|c|c|}
\hline Genotypes & Agro climatic zones/situation & Genotypes & Agro climatic zones/situation \\
\hline VGG4 & Karnataka & PM5 & Madhya Pradesh \\
\hline PS 16 & Karnataka, Assam, Delhi, Bihar, & HUM1 & $\begin{array}{l}\text { Karnataka, Gujarat, Madhya } \\
\text { Pradesh, Tamilnadu, }\end{array}$ \\
\hline K851 & $\begin{array}{l}\text { Andhra Pradesh, Himachal Pradesh, } \\
\text { Rajasthan }\end{array}$ & AKM8803 & Maharashtra \\
\hline PB1 & Karnataka, Maharashtra, Tamil nadu & KKM4 & Karnataka \\
\hline TAP7 & Maharashtra & KM15 & Karnataka cultivars \\
\hline TARM2 & Maharashtra (ikisan) & KM16 & Karnataka cultivars \\
\hline SML668 & $\begin{array}{l}\text { Himachal Pradesh, Punjab, Haryana, } \\
\text { Western Uttar Pradesh. }\end{array}$ & KM 28 & Karnataka cultivars \\
\hline PSA9591 & Haryana, Maharashtra & KM 30 & Karnataka cultivars \\
\hline PM1 & $\begin{array}{l}\text { North western zone, Haryana. } \\
\text { (ikisan) }\end{array}$ & KM31 & Karnataka cultivars \\
\hline HUM12 & North eastern plains & KM 39 & Karnataka cultivars \\
\hline SML348 & $\begin{array}{l}\text { Punjab, Haryana, Western Uttar } \\
\text { Pradesh. }\end{array}$ & KM 45 & Karnataka cultivars \\
\hline LM182 & Haryana & KM 46 & Karnataka cultivars \\
\hline KKM3 & $\begin{array}{c}\text { Punjab, Haryana, Western Uttar } \\
\text { Pradesh. }\end{array}$ & KM 47 & Karnataka cultivars \\
\hline MAVT836 & Haryana, Maharashtra & KM 48 & Karnataka cultivars \\
\hline NP36 & Maharashtra & KM 51 & Karnataka cultivars \\
\hline MDV3156 & Punjab & KM 59 & Karnataka cultivars \\
\hline PM103 & $\begin{array}{c}\text { Orissa, Andhra Pradesh, Assam, } \\
\text { Karnataka }\end{array}$ & KM 60 & Karnataka cultivars \\
\hline M108 & $\begin{array}{c}\text { Punjab, Haryana, Western Uttar } \\
\text { Pradesh. }\end{array}$ & KM 49 & Karnataka cultivars \\
\hline NDM1 & $\begin{array}{l}\text { Gujrat, Madhya Pradesh, Tamilnadu, } \\
\text { Maharashtra. (ikisan) }\end{array}$ & KM 70 & Karnataka cultivars \\
\hline PM2 & Orissa, Andhra Pradesh, Assam & KM 78 & Karnataka cultivars \\
\hline PRATHAP & $\begin{array}{l}\text { Orissa, Andhra Pradesh, Assam, } \\
\text { Karnataka }\end{array}$ & KM 4 & Karnataka cultivars \\
\hline SML134 & Punjab & KM 3 & Karnataka cultivars \\
\hline PUSA9072 & Maharashtra & KM 10 & Karnataka cultivars \\
\hline PDM11 & Uttar Pradesh & MDV31 & \\
\hline KAPORGAON & $\begin{array}{l}\text { Orissa, Andhra Pradesh, Assam, } \\
\text { Karnataka }\end{array}$ & KKM3 & Karnataka \\
\hline PUSA9531 & Bihar, Gujarat. & PRATHAP & $\begin{array}{l}\text { Andhra Pradesh, Assam, } \\
\text { Karnataka, Odissa }\end{array}$ \\
\hline PUSA VISHAL & Uttar Pradesh, Madhya Pradesh. & PDM11 & $\begin{array}{c}\text { Andhra Pradesh, Assam, } \\
\text { Karnataka }\end{array}$ \\
\hline
\end{tabular}


Table. 2 List of RGA primers used in the study

\begin{tabular}{|c|c|c|c|}
\hline Sl. No & Name & Forward sequence & Tm value \\
\hline \multirow[t]{2}{*}{1} & XLRR-F & CCGTTGGACAGGAAGGAG & $47^{\circ} \mathrm{C}$ \\
\hline & XLRR-R & CCCATAGACCGGACTGTT & \\
\hline \multirow[t]{2}{*}{2} & CLRR-F & TTTTCGTGTTCAACGACG & $43^{\circ} \mathrm{C}$ \\
\hline & CLRR-R & TAACGTCTATCGACTTCT & \\
\hline \multirow[t]{2}{*}{3} & RLRR-F & CGCAACCACTAGAGTAAC & $47^{\circ} \mathrm{C}$ \\
\hline & RLRR-R & ACACTGGTCCATGAGGTT & \\
\hline \multirow[t]{2}{*}{4} & NLRR-F & TAGGGCCTCTTGCATCGT & $43^{\circ} \mathrm{C}$ \\
\hline & NLRR-R & TATAAAAAGTGCCGGACT & \\
\hline \multirow[t]{2}{*}{5} & NPLOOP-F & TCAATTAATGTTTGAGTTATTGTA & $43^{\circ} \mathrm{C}$ \\
\hline & Nkin2-R & GTAACTAAGGATAGA & \\
\hline \multirow[t]{2}{*}{6} & Pto kin1-F & GCATTGGAACAAGGTGAA & $43^{\circ} \mathrm{C}$ \\
\hline & Pto kin2-R & AGGGGGACCACCACGTAG & \\
\hline \multirow[t]{2}{*}{7} & Pto kin3-F & TAGTTCGGACGTTTACAT & $44^{\circ} \mathrm{C}$ \\
\hline & Pto kin4-R & AGTGTCTTGTAGGGTATC & \\
\hline \multirow[t]{2}{*}{8} & NBS-F & GGAATGGGNGGNGTNGGNAARAC & $44^{\circ} \mathrm{C}$ \\
\hline & NBS-R & YCTAGTTGTRAYDATDAYYYTRC & \\
\hline \multirow[t]{2}{*}{9} & RLK-F & GAYGTNAARCCIGARAA & $46^{\circ} \mathrm{C}$ \\
\hline & RLK-R & TCYGGYGCRATRTANCCNGGITGICC & \\
\hline \multirow[t]{2}{*}{10} & S1-F & GGTGGGGTTGGGAAGACAACG & $46^{\circ} \mathrm{C}$ \\
\hline & AS1-R & CAACGCTAGTGGCAATCC & \\
\hline \multirow[t]{2}{*}{11} & S2-F & GGIGGIGTIGGIAAIACIAC & $46^{\circ} \mathrm{C}$ \\
\hline & AS3-R & IAGIGCIAGIGGIAGICC & \\
\hline \multirow[t]{2}{*}{12} & PtoFen-S-F & ATGGGAAGCAAGTATTCAAGGC & $45^{\circ} \mathrm{C}$ \\
\hline & PtoFen-AS-R & TTGGCACAAAATTCTCATCAAGC & \\
\hline \multirow[t]{2}{*}{13} & XLRR-INV1-F & TTGTCAGGCCAGATACCC & $47^{\circ} \mathrm{C}$ \\
\hline & XLRR-INV2-R & GAGGAAGGACAGGTTGCC & \\
\hline \multirow[t]{2}{*}{14} & CLRR-INV1-F & GCAGCAACTTGTGC & $47^{\circ} \mathrm{C}$ \\
\hline & CLRR-INV2-R & TCTTCAGCTATCTGC & \\
\hline \multirow[t]{2}{*}{15} & NLRR-INV1-F & TGCTACGTTCTCCGGG & $45^{\circ} \mathrm{C}$ \\
\hline & NLRR-INV2-R & TCAGGCCGTGAAAAATAT & \\
\hline \multirow[t]{2}{*}{16} & Pto-kin1IN-F & AAGTGGAACAAGGTTACG & $45^{\circ} \mathrm{C}$ \\
\hline & Pto-kin2IN-R & GATGCACCACCAGGGGG & \\
\hline \multirow[t]{2}{*}{17} & S1-INV-F & GCAACAGAAGGGTTGGGGTGG & $45^{\circ} \mathrm{C}$ \\
\hline & AS1-INV-R & CCTAACGGTGATCGCAAC & $45^{\circ} \mathrm{C}$ \\
\hline \multirow[t]{2}{*}{18} & S2-INV-F & CAICAIAAIGGITGIGGIGG & \\
\hline & AS3-INV-R & CCIGAIGGIGAICGIG & \\
\hline \multirow[t]{2}{*}{19} & wlrk-S-F & GAAAGATGAGTAAATTACTTG & $45^{\circ} \mathrm{C}$ \\
\hline & wlrk-AS-R & TGAGGGTCAGGCATGCAG & \\
\hline
\end{tabular}




\begin{tabular}{|c|c|c|c|}
\hline \multirow[t]{2}{*}{20} & Cre3Ploop-F & GCGGGTCTGGGAAATCTACC & $46^{\circ} \mathrm{C}$ \\
\hline & Cre3-k3-R & CTGCAGTAAGCAAAGCAACG- & \\
\hline \multirow[t]{2}{*}{21} & Cre3LR-F & CACACACTCGTCAGTCTGCC & $45^{\circ} \mathrm{C}$ \\
\hline & Cre3LR-R & CAGGAGCCAAAAATACGTAAG & \\
\hline \multirow[t]{2}{*}{22} & Xa1NBS-F & GGCAATGGAGGGATAGG & $46^{\circ} \mathrm{C}$ \\
\hline & Xa1NBS-R & CTCTGTATACGAGTTGTC & \\
\hline \multirow[t]{2}{*}{23} & Xa1LR-F & СТCАСТСТССТGAGAAAATTAC & $44^{\circ} \mathrm{C}$ \\
\hline & Xa1LR-R & GAGATTGCCAAGCAATTGC & \\
\hline \multirow[t]{2}{*}{24} & RGA-1-F-CGa & AGTTTATAATTCGATTGC & $49^{\circ} \mathrm{C}$ \\
\hline & RGA-1-R & ACTACGATTCAAGACGTCCT & \\
\hline \multirow[t]{2}{*}{25} & RGA-1-F-CGb & AGTTTATAATTCGATTGCT & $49^{\circ} \mathrm{C}$ \\
\hline & RGA-1-R & ACTACGATTCAAGACGTCCT & \\
\hline \multirow[t]{2}{*}{26} & RGA-1-F-TG & AGTTTATAATTTGATTGCT & $49^{\circ} \mathrm{C}$ \\
\hline & RGA-1-R & ACTACGATTCAAGACGTCCT & \\
\hline \multirow[t]{2}{*}{27} & MtB331-F & GGCTTCCTGATGCTGGTTAG & $48^{\circ} \mathrm{C}$ \\
\hline & MtB331-R & ACAAGCAGGTTGGACACACA & \\
\hline \multirow[t]{2}{*}{28} & MtB99-F & CTTGGCAAAATGTCAACTCT & $47^{\circ} \mathrm{C}$ \\
\hline & MtB99-R & GGAAAGGGGTTAGGTGAGTA & \\
\hline \multirow[t]{2}{*}{29} & h2_119h6a-F & CGCACGAGTTGGATATGATG & $48^{\circ} \mathrm{C}$ \\
\hline & h2_13m22a-R & CGTCGCACGAGTTTACTGAT & \\
\hline \multirow[t]{2}{*}{30} & h2_13m22a-F & TCAAACTCAAGCCACCACAA & $47^{\circ} \mathrm{C}$ \\
\hline & h2_13m22a-R & GCTCGAGTCATGGAGGGTAA & \\
\hline \multirow[t]{2}{*}{31} & VuRS01N7V & GAACGGTGAAGATTGGAATTTG & $49^{\circ} \mathrm{C}$ \\
\hline & & CTATGACCGAGTGTTGCATGAT & \\
\hline \multirow[t]{2}{*}{32} & VuRS01J7V & TCTCCAAAACCAGAGAGTTGC & $48^{\circ} \mathrm{C}$ \\
\hline & & GGGCAGAATTGGAAATTTGA & \\
\hline \multirow[t]{2}{*}{33} & VuRS01N9V & GCCAATTCAGCACAAGGTTT & $48^{\circ} \mathrm{C}$ \\
\hline & & TAGGTGGAGGATGTGCATTG & \\
\hline \multirow[t]{2}{*}{34} & VuRS02A2R & CCAGCGTAGTGATGTTCTTGAG & $46^{\circ} \mathrm{C}$ \\
\hline & & GCAACCCTTGATAGCTTATGGA & \\
\hline \multirow[t]{2}{*}{35} & VuRS02F16V & CCAATGCCTTGAGGATTAAAA & $51^{\circ} \mathrm{C}$ \\
\hline & & CGGTCTAAGTCGGTCATGAAG & \\
\hline \multirow[t]{2}{*}{36} & VuRS01A03R & TGAGCAATCTTTCCCCAATC & $53^{\circ} \mathrm{C}$ \\
\hline & & CCACGCTCTCTCACCTCTCT & \\
\hline \multirow[t]{2}{*}{37} & VuRS01G15R & GCAGTGCACTCCAATTCCTA & $51^{\circ} \mathrm{C}$ \\
\hline & & CGCCATTAAGCATAGCACAC & \\
\hline \multirow[t]{2}{*}{38} & VuRS01G18V & GGATGACAACGAGGCTTTATTC & $45^{\circ} \mathrm{C}$ \\
\hline & & TACTGGAGTGGACAGAGTGTGG & \\
\hline \multirow[t]{2}{*}{39} & VuRS01G04R & CTGTTCTGCTTGTTGGTTTTCA & $47^{\circ} \mathrm{C}$ \\
\hline & & AGGTTTGTTGATCGTCAGGAAG & \\
\hline
\end{tabular}




\begin{tabular}{|c|c|c|c|}
\hline \multirow[t]{2}{*}{40} & VuRS01J11R & TCCGTGATTTTACGCCTTTC & $47^{\circ} \mathrm{C}$ \\
\hline & & AAAATAGGTTTAATTGGAACGGACT & \\
\hline \multirow[t]{2}{*}{41} & VuRS01K16R & TACCCACGGGTACGGGTATT & $47^{\circ} \mathrm{C}$ \\
\hline & & TGAAGAATGAAGACAGAAGACAAGA & \\
\hline \multirow[t]{2}{*}{42} & VuRS01L21R & TGAGTTAGCCGTTTTGGGTA & $47^{\circ} \mathrm{C}$ \\
\hline & & GCCTGGAATTGCAAAAATGT & \\
\hline \multirow[t]{2}{*}{43} & VuRS02H07V & CTCTTCGTTATCCCCTCTGCTA & $47^{\circ} \mathrm{C}$ \\
\hline & & CAGGTCTCTGGTGCTCTACCTT & \\
\hline \multirow[t]{2}{*}{44} & VuRS02L24R & TGGCGAAATTGTACTAAGCAAG & $50^{\circ} \mathrm{C}$ \\
\hline & & AAGGGGTTTAGAAAAGAGGGTG & \\
\hline \multirow[t]{2}{*}{45} & VuRS03A23V & CAGGCATGCAAGCTTCTCTT & $47^{\circ} \mathrm{C}$ \\
\hline & & CAATTCTAGCCGGGTAAAGG & \\
\hline \multirow[t]{2}{*}{46} & VuRS03G17V & CAGAATACACGAAACGAAAGTG & $47^{\circ} \mathrm{C}$ \\
\hline & & CCGATTGGACAGTTTAAGAAGA & \\
\hline \multirow[t]{2}{*}{47} & VuRS03O19V & TACGTGTGAAAATTGCTTGACC & $51^{\circ} \mathrm{C}$ \\
\hline & & TAGAATTGGGAGATTTGGAACG & \\
\hline \multirow[t]{2}{*}{48} & VuRS01P18V & TTTGGATTCTTTCCCGTGTT & $47^{\circ} \mathrm{C}$ \\
\hline & & ATTCTTGGCTGACTCGCAAT & \\
\hline \multirow[t]{2}{*}{49} & VuRS01P23V & TTTATGTTTTGAGAATCATTGCAG & $47^{\circ} \mathrm{C}$ \\
\hline & & CGTGGATTTTGAAACCTCCATA & \\
\hline \multirow[t]{2}{*}{50} & VuRS02B24R & GTGGCTTGATGAGGATATGAAA & $47^{\circ} \mathrm{C}$ \\
\hline & & GGAGAAGTGTATTTGTTGTTGAGAG & \\
\hline \multirow[t]{2}{*}{51} & VuRS02F19V & GGTGGAACAGACGACATGAA & $47^{\circ} \mathrm{C}$ \\
\hline & & GTGATCTCTTTGGCCCATGT & \\
\hline \multirow[t]{2}{*}{52} & VuRS02H06R & AGTCAACAAGGGAAAGCAAGAG & $48^{\circ} \mathrm{C}$ \\
\hline & & CCATGAGTCTGTGAGTTTGCAT & \\
\hline \multirow[t]{2}{*}{53} & VuRS02M19V & GAACCCACAACCCTGAAATG & $48^{\circ} \mathrm{C}$ \\
\hline & & TGAGAGGACTTGGGTTCGAG & \\
\hline \multirow[t]{2}{*}{54} & VuRS02M22R & CACAAGCCCTAGCACTCCTC & $48^{\circ} \mathrm{C}$ \\
\hline & & CTAGTTTTGCCCCCTGTTTG & \\
\hline \multirow[t]{2}{*}{55} & RGA1-F & GGNTGNATNGGNTANGANCAN & $48^{\circ} \mathrm{C}$ \\
\hline & RGA1-R & GANCTNTGNAANGANATNAAN & \\
\hline
\end{tabular}


Table.3 Number of alleles, allele frequency, gene diversity, polymorphic information content for fifty four genotypes of green gram

\begin{tabular}{|l|c|c|c|c|c|}
\hline Marker & $\begin{array}{c}\text { Frequency of } \\
\text { abundant Allele }\end{array}$ & $\begin{array}{c}\text { Sample } \\
\text { Size }\end{array}$ & Allele No & $\begin{array}{c}\text { Gene } \\
\text { Diversity }\end{array}$ & PIC \\
\hline XLRR & 0.596 & 54 & 3 & 0.493 & 0.463 \\
\hline Pto kin1 & 1 & 54 & 1 & 0 & 0 \\
\hline NBS & 1 & 54 & 1 & 0 & 0 \\
\hline S2 & 1 & 54 & 1 & 0 & 0 \\
\hline S1-INV & 0.5 & 54 & 3 & 0.664 & 0.61 \\
\hline WIrk-S & 1 & 54 & 1 & 0 & 0 \\
\hline Cre3Ploop & 1 & 54 & 1 & 0 & 0 \\
\hline Xa1LR & 1 & 54 & 1 & 0 & 0 \\
\hline RGA-1CGa & 1 & 54 & 1 & 0 & 0 \\
\hline RGA-1CGb & 1 & 54 & 1 & 0 & 0 \\
\hline RGA-1TG & 0.592 & 54 & 2 & 0.4753 & 0.36 \\
\hline MtB331 & 1 & 54 & 1 & 0 & 0 \\
\hline MtB99 & 0.59 & 54 & 2 & 0.482 & 0.366 \\
\hline h2119h6a & 1 & 54 & 1 & 0 & 0 \\
\hline h213m22a & 1 & 54 & 1 & 0 & 0 \\
\hline S1 & 0.518 & 54 & 3 & 0.55 & 0.46 \\
\hline CLRR-INV1 & 1 & 54 & 1 & 0 & 0 \\
\hline Pto-kin1IN & 0.49 & 54 & 3 & 0.59 & 0.51 \\
\hline VuRS01N7V & 1 & 54 & 1 & 0 & 0 \\
\hline VuRS01N9V & 1 & 54 & 1 & 0 & 0 \\
\hline VuRS02A2R & 1 & 54 & 1 & 0 & 0 \\
\hline VuRS01A03R & 1 & 54 & 1 & 0 & 0 \\
\hline VuRS01G04R & 1 & 54 & 1 & 0 & 0 \\
\hline VuRS01L21R & 1 & 54 & 1 & 0 & 0 \\
\hline VuRS02H07V & 1 & 54 & 1 & 0 & 0 \\
\hline VuRS03A23V & 1 & 54 & 1 & 0 & 0 \\
\hline VuRS03G17V & 1 & 54 & 1 & 0 & 0 \\
\hline VuRS03019V & 1 & 54 & 1 & 0 & 0 \\
\hline VuRS02H06R & 1 & 54 & 1 & 0 & 0 \\
\hline VuRS02F16V & 0.53 & 54 & 3 & 0.614 & 0.54 \\
\hline VuRS02L24R & 1 & 54 & 1 & 0 & 0 \\
\hline VuRS02B24R & 1 & 54 & 1 & 0 & 0 \\
\hline & & & & & \\
\hline
\end{tabular}


Fig.1 PCR amplification generated by MTB99 marker in mungbean in 3\% agarose gel electrophoresis

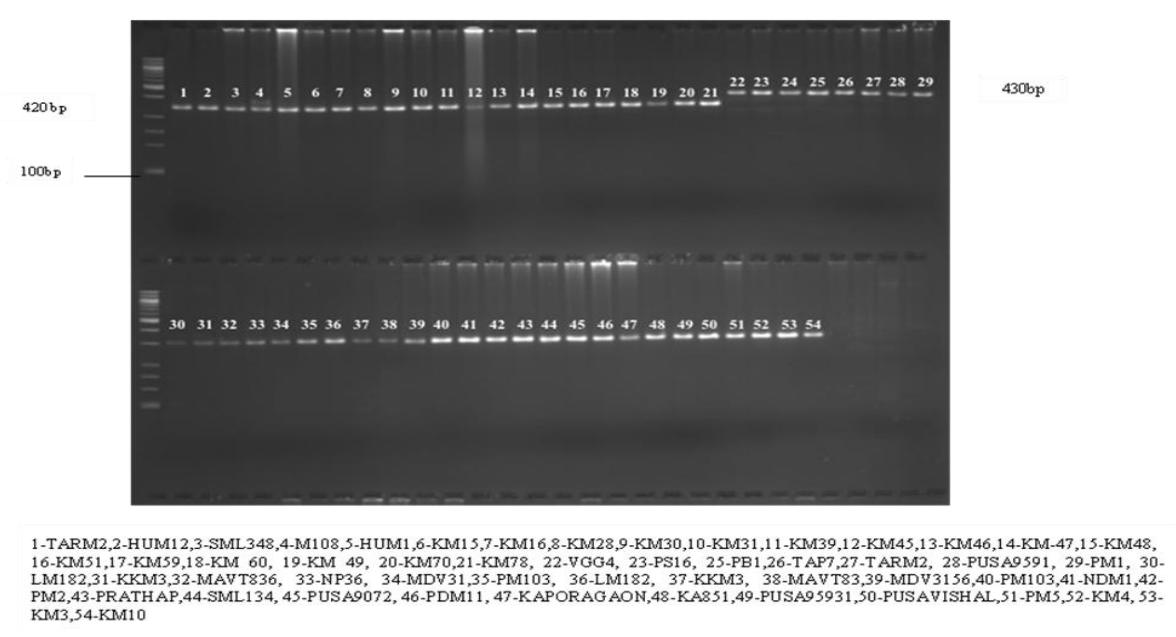

Out of 32 markers seven markers generated polymorphic amplicones and 25 markers generated monomorphic amplicons. Total of 40 alleles were generated with an average of 2 alleles per markers for the genotypes evaluated. Majority of markers amplified one allele per marker. Number of alleles ranged from 1 to 3 . The allele frequency was least for Pto-kin1IN (0.49).The Polymorphic Information Content (PIC) (Table 3) of individual loci ranged from 0.00 to 0.61 with a mean value of 0.07 in green gram, the highest value (0.61) belonged to S1-INV followed by VuRS02F16V (0.54).

Out of the 55 markers used, 32 markers generated amplification. Out of the 32 primers amplified, only 7 found to be polymorphic. However, these 7 polymorphic markers collectively yielded 15 in mungbean, with an average of 2.1polymorphic alleles per locus respectively, which was comparable to 3.9 alleles perlocus obtained by Gupta and Gopalakrishna (2010) in a study using EST derived SSR markers in cowpea. These results were also comparable to studies done using genomic SSR markers in Vigna species including cowpea (4.6 alleles per locus; Li et al., 2001), urdbean (4.1 alleles per locus; Gupta and Gopalakrishna 2009) and azuki bean (4 alleles perlocus; Wang et al., 2004). As the resistant gene homologues are derived from the NBS-LRR disease resistant motifs it offers resistant to fungal, bacterial and viral pathogens (Gupta and Gopalakrishna, 2010). Number of reasons could be attributed to his. Firstly, the RGH's are generally considered less polymorphic as compared to genomic SSR markers (Eujayl et al., 2001; Gupta et al., 2003). As the RGH markers are conserved across generations and also across species, the degree of polymorphism that can be expected using them is very low. Secondly, low polymorphism obtained may be because of the use of makers belonging to cowpea, chickpea, red gram and Medicago (Gupta and Gopalakrishna, 2010). In 2017 Sagi et al., studied the Genetic Analysis of NBS-LRR Gene Family and their Expression Profiles in Chickpea in response to ascochyta blight infection. Recently Wu et al., 2017 reported the 178 NBS-LRR-type genes and 145 partial genes were associated with Anthracnose and Common Bacterial Blight in the Common Bean.

Not many markers are developed specifically for mungbean; hence researchers use the markers that are designed from cowpea, common bean and soya bean extensively in 
these two crops. Though recently, SSR markers have been developed from mungbean, the number of these SSR's is still very limited. The genotypes TARM 2, HUM12, SML348, HUM1 VGG4, PS16 PB1, TAP 7 KM 15 KM 16 KM 30 are found resistance to mungbean yellow mosaic virus these genotypes can be used as a resistance source for further resistance breeding programme

\section{Acknowledgement}

Authors are grateful to Head Division of institute of agriculture biotechnology Dharwad, for supporting and giving valuable suggestion throughout the study. Head of genetics and plant breeding for providing the field and lab to carried out experiments, DBTJNU for providing financial support in my degree programme.

\section{References}

Eujayl, I., Sorrells, N.E., Baum, M., Wolters, P. and Powell, W., 2001, Assessment of genotype variation among cultivated durum wheat based on ESTSSR's and genomic SSR's. Euphytica, 119(1/2):39-43.

Gupta, P. K., Rustgi, S, Sharma, S., Singh, R., Kumar, N. and Balyan, H.S., 2003, Transferable EST, SSR markers for the study of polymorphism and genetic diversity in bread wheat. Mol. Gen. Genom., 270:315-323.

Gupta, S. K. and Gopalakrishna, T., 2010, Development of unigene - derived SSR markers in cowpea (Vigna ungiculata) and their transferability to other Vigna species. Genome, 53: 508523.

Huang, L. and Gill, B. S., 2001, An RGA-like marker detects all known Lr21 leaf rust resistant gene family members in Aegilopstauschii and wheat, Theor.
Appl. Genet., 103:1007-1013

Kanazin, V., Marek, L. F. and Shoemaker, R. C., 1996, Resistant gene analogs are conserved and clustered in soyabean, Proc. Natl. Acad. Sci USA, 93: $11746-$ 11750.

Kaur G., Arunabh Joshi, Devendra Jain. SSRMarker assisted evaluation of Genetic Diversity in Mungbean (Vigna radiata (L.) Wilcezk) genotypes, journal of Brazilian Archives of Biology and Technology, 61: 1678-4324

Li C. D., Fatokun, C. A., Ubi, B., Singh, B.B. and Scoles, G.J., 2001, Determining genetic similarities and relationships among cowpea breeding lines and cultivars by microsatellite markers. Crop Sci., 41: 189-197.

Liu, K. and Muse, S. V., 2005, Power marker: Integrated analysis environment for genetic marker data. Bioinformatics, 21: 2128-2129.

Nair R., Roland S., Warwick Easdown and Andreas E. 2014. Legume improvement program at AVRDC The World Vegetable Centre: Impact and future prospects, Ratar. Povrt. 51:1 55-61.

Paul, P. C., Biswas, M. K., Mandal, D. and Pal, P., 2013, Studies on host resistant of mungbean against mungbean yellow mosaic virus in the agroecological condition of lateritic zone of West Bengal, Bioscan, 8(2): 583587.

Saghai, Maroof, M.A., Soliman, K.M., Jorgenson, R. and Allard, R.W., 1984, Ribosomal DNA spacer length polymorphism in barley: Mendelian inheritance, chromosomal locations and population dynamics. Proc. Natl. Acad. Sci. USA. 81:8014-8018.

Sagi M, S., Amit A. Deokar and Bunyamin Taran. 2017. Genetic Analysis of NBS-LRR Gene Family in Chickpea and Their Expression Profiles in 
Response to Ascochyta Blight Wu Jing, Jifeng Zhu, Lanfen Wang Infection. Frontiers in Plant Science, 8:838.

Singh, D. P., 1980, Inheritance of resistant to yellow mosaic virus in urdbean (Vigna mungo (L.) Hepper). Theor. Appl. Genet., 57: 233-235. and Shumin Wang. 2017. GenomeWide Association Study Identifies NBS-LRR-Encoding Genes Related with Anthracnose and Common Bacterial Blight in the Common Bean. Frontiers in Plant Science, 8:1398

Wang, X. W., Kaga, A., Tomooka, N. and Vaughan, D.A., 2004. Development of SSr markers by a new method in plants and their application to gene flow studies in Azuki bean [Vigna angularis (Willd.) Ohwi and Ohashi]. Theor. Appl. Genet., 109 (2): 352-360.

Yan, G. P., Chen, X. P., Line, R. F. and Wellings, C. R., 2003, Resistant-gene analog polymorphism markers cosegregating with the Yr5 gene for resistant to wheat stripe rust, Theor. Appl. Genet., 106: 636-643.

\section{How to cite this article:}

Jyothi, N., B. R. Patil, Ramesh Bhat and Lokesh kumar, B. M. 2020. NBS-LLR Marker Assisted Screening of Resistance Genotypes for Mungbean Yellow Mosaic Virus (MYMV) in Mungbean (Vigna radiata (L.) Wilcezk) Genotypes. Int.J.Curr.Microbiol.App.Sci. 9(08): 28762886. doi: https://doi.org/10.20546/ijcmas.2020.908.323 